\title{
Biopolymer pullulan (polysaccharide) with potential as a edible coating \& film and their application in food additives and drug
}

\section{Pullulan}

The biopolymer is generally considered to be soluble in water. Its production is obtained from a variety of combinations of microorganisms, which have new and unique physical properties. Because of these properties, Thus the polymers have been utilized in a wide spectrum of pharmaceuticals, food, and environmental applications in food, pharmaceutical and other companies because they contain emulsifiers, Stabilizers, binders, crystallizing agents, coagulants, lubricants, natural films, thickening agents, suspending agents and these technological properties are provided by these new polymeric materials by adding them in mixtures, components, compounds, etc. ${ }^{1}$ A mixture of polymers is prepared by physical homogenizing of two polymers or more as it has a property superior to the use of one of the single polymer and pullulan is one of the polymers obtained from the fermented medium of the yeast aureobasidium pullulans, a polymer composed of polysaccharides, which consists of maltotriose units bind to each other by an alpha bond $\alpha(1 \rightarrow 4)$ glucose as it contains three glucose units in successive maltotriose are bound together by an $\alpha$. bond $(1 \rightarrow 6)$ glycosidic bond $(\alpha-1,6$ glycosidic bond). The average molecular weight of pullulan is $(0-200)$ glucose units. It is a natural substance found in the cell
Volume II Issue I - 202 I

\section{Hosam El Din Aboul anean}

Food Engineering and Packaging Department, Food Technology Research Institute (FTRI), Agriculture Research Center (ARC), Egypt

Correspondence: Hosam El din Aboul anean, Food Engineering and Packaging Department, Food Technology Research Institute (FTRI), Agriculture Research Center (ARC), Giza, Egypt, Tel 01 I I 2667765 - 01018842643 , Fax (+202)35684699, Email Hosam.ftri@yahoo.com

Received: November 26, 202I | Published: December 14, 2021

walls. It is commercially produced from the fermentation process of carbohydrates (sugar and starch) by yeast or produces enzymatic n $\left(\mathrm{C}_{6} \mathrm{H}_{12} \mathrm{O}_{5}\right){ }^{2}$ Figure $1 \&$ Figure 2, illustrates the structure of the pullulan and the basic bonds in the pullulan and the sites of water enzymatic hydrolysis. ${ }^{3}$

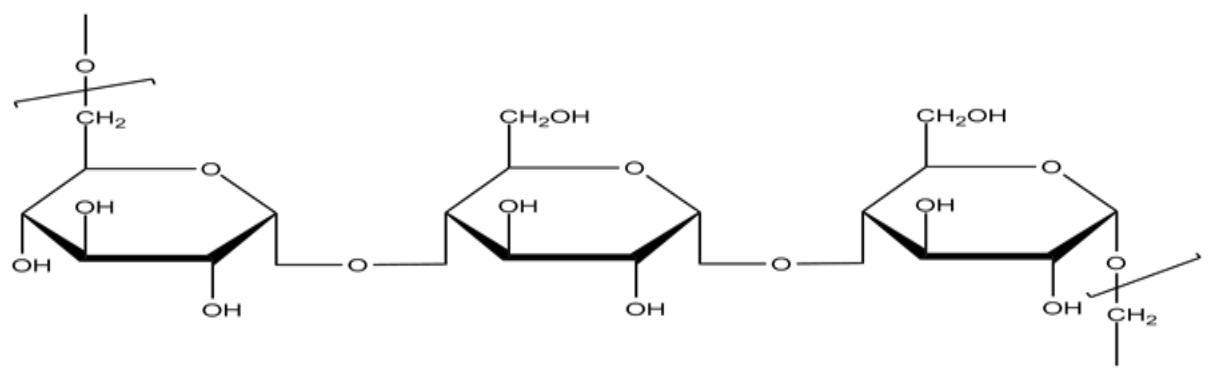

Figure I Structure of pullulan.
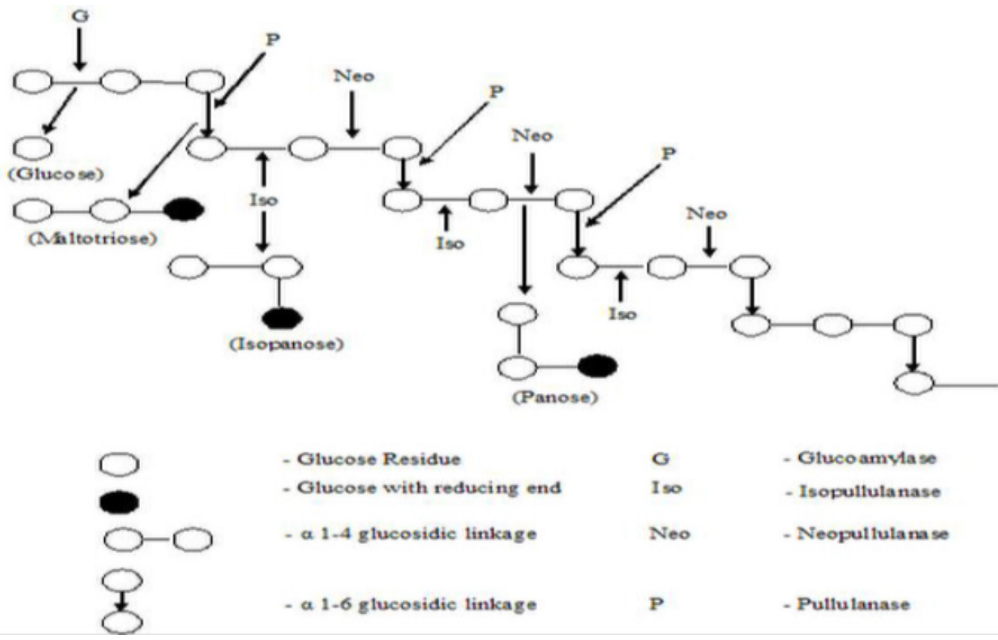

Figure 2 Basic bonds in the pullulan and the sites of water enzymatic hydrolysi. 


\section{Pullulan usage}

a. A natural polysaccharide polymer, the fermentation process of aureobasidium pullulans, as the aura of the substance contains the black pigment melanin. ${ }^{4}$

b. It is used in manufacturing and pharmaceuticals and various biomedical applications, as one of its characteristics is being a polymer of others, and the case, and it does not occur. Displacements of nanoparticles as this clinic can express its merits. ${ }^{5,6}$

c. It is used in tissue engineering, wound healing, and diagnostic applications such as perfusion, receptors, defining lymph nodes, and vascular compartment imaging to bind to $\alpha(1 \rightarrow 4)$ glucose and $\alpha(1 \rightarrow 6)$ glucan binding in pullulan. This polymer also confers distinctive physical properties including adhesion. From the fiber industry.

d. It is a tasteless polymer that is used in inactive ingredients and colors, a mouth freshener, and antiseptic such as Listerine Cool Mint. It is also used in cleansing the face and skincare and works as an aid in regulating blood sugar.

e. Combining pullulan product with a water-soluble low molecular weight protein can inhibit antiviral activity and regulate the system, used to maintain the biological efficacy of the drug.

f. 6-It is also used in the production of capsules for solid drug formulations. Pullulan protects the covered products and the materials contained in them from the harmful effects of air, moisture, and light.

g. Its source is considered industrial as polymeric materials, which have gradually become economically competitive advantage with the natural gum made from sea algae and other plants, and the hydrocolloid gum, which is a description of sugars where viscosity and gelatinization are used. ${ }^{8}$

h. It has been proven that it is an adhesive foam with good properties when it dissolves in water and a solution of waterbased food colloids is formed, which has strong properties as it reduces friction between the enzyme and the chitosan activity material loaded on it and thus reduces the volume of food transport in the intestine as a result of increased viscosity and thus can be used in weight control for obese patients, pullulan product plays an important role in inhibiting the activity of enzymes that digest carbohydrates, as this activity appears against enzymes such as invertase, amylase, glucose oxidase, $\beta$-glusosidase, and frutosyltransferase analyzer for sugars and starches, and small amounts of a proteolytic enzyme. ${ }^{9}$

i. Pullulan product is also used in the food industry, such as thickening materials, emulsifiers, stabilizers, and water colloids, which slow down the digestion of starch due to the formation of a physical excellent barrier that limits digestive enzymes. ${ }^{10}$

j. It can also help in the production of non-traditional natural films, extending the shelf life of the product, and in food fortification as a food additive. ${ }^{11}$

k. Where these pullulan product substances prevent the growth of microbes and oxygen and maintain the improvement of the quality and stability of the film for coating the grains and improve the sensory, physical and mechanical.

1. Pullulan product is also an effective carrier of public health safety and antioxidants, flavoring, or antimicrobial additives that have nutritional value with the ability to eat, digest, absorb and benefit the human body from them.

m. We can point out that this substance may be useful for application in the field of food and pharmaceutical industry and has a simple production technology as its production cost is low and thus reduces the economic cost of the product. ${ }^{12}$

n. Hence, the use of alternative and harmless sources for human health and the environment is represented in the use of pullulan products to manufacture a natural food film as healthy and safe alternatives to traditional packaging materials prepared from plastic materials and other packaging materials harmful to foodstuffs, human health, and the environment, so that foodstuffs can be wrapped and consumed Direct as these natural membranes are safe and harmless to human health. ${ }^{13}$

\section{Summary}

We conclude that the pullulan product is a natural substance produced from polymers of polysaccharides and is commercially produced through the fermentation process of carbohydrates (sugar and starch) by yeast or produced enzymatically. The pullulan is obtained from the fermentation process with aureobasidium pullulans, producing polysaccharides polymers that contain in their composition units of maltose and one of the glucose units in maltotriose, which is linked to each other by its n-glucosidic bond $\left(\mathrm{C}_{6} \mathrm{H}_{12} \mathrm{O}_{5}\right) .{ }^{2}$ This polymer is characterized as being non-toxic, non-allergic, non-carcinogenic, and does not cause genetic mutations through gene delivery. It is used as a mouth freshener and antiseptic, and it competes with natural gums and hydrocolloids, which have strong properties that disrupt friction. Between the enzyme and the active substance carried on it, and thus reduces the volume of food transport in the intestine due to an increase in its viscosity, and therefore it can be used in weight control for obese patients, as it slows down the digestion of starch due to the formation of a physical barrier that limits the effect of digestive enzymes and also inhibits the activity of enzymes that work on the digestion of carbohydrates and is used as a factor and assistant in the treatment of regulating blood sugar and an effective carrier of public health safety. It is also of high nutritional value with the ability to eat, digest, absorb and benefit the human body from it. We can point out that this substance is useful for application in the manufacture of tablets of microparticles loaded with pandrones as a means of transporting small particles of nanoparticles where these drugs can cross the bloodbrain barrier for the purpose of treating neurological disorders. ${ }^{13,14}$ It also has many applications in the food industry such as thickeners, emulsifiers, stabilizers, and water colloids, where it can slow down the digestion of starch due to the formation of a physical barrier that limits the effect of the digestive enzymes of pullulan product because of its role which is to inhibit the activity of enzymes that digest carbohydrates, as this activity appears against enzymes such as invertase, amylase, glucose oxidase, $\beta$-glusosidase, frutosyltransferase that break down carbohydrates and sugars, and small amounts of the enzyme proteolytic enzyme. From this, it becomes clear to us that the pullulan product is of high nutritional value with its ability to eat, digest, absorb and benefit the human body from it, as it has a simple production technology as the cost of production is low and therefore the economic cost of the product is reduced. ${ }^{3}$

\section{Article significance}

The economic return from the article: Nano biopolymers will be produced to benefit from them in improving the properties of natural food membranes, as well as benefiting from them in the field of medicines and as a food supplement due to their multiple natural benefits, as the cost of these materials is much lower than the industrial 
materials used for the same purpose, which is characterized by its high price because they are industrial materials and there are serious health damages in using them, as these industrial materials cause types of allergies that accumulate inside the human body and also lead to the occurrence of carcinogenic diseases in the long run. It is worth noting that these naturally produced polymeric materials are produced in an economical way, with low costs, with many advantages for consumer health, nutritional value, and harmless to human health and the environment with their ability to digest and absorb the benefits to the human body from them compared to high-priced industrial materials that pollute the environment when disposed of. ${ }^{15,16}$

\section{Acknowledgments}

None.

\section{Conflicts of interest}

The author states there are no conflicts of interest.

\section{Funding}

None.

\section{References}

1. Carolan G, Catley BJ, Mcoungal F J. The location of tetrasaccharide units in pullulan. Carbohyd Res. 1983;114:237-243.

2. Aboul-Anean H El Din. Biopolymer product from pullulan material (polysaccharide) used for natural film \& coa'ngs in food preserva'on. Archives of Nutri'on and Public Health. 2021;3(1):1-14.

3. Youki Suzuki, Yutaka Kataoka. Hiroaki MatsuiElectrical properties of composite cyanoethylated pullulan film. J Wood Sci. 2003;49:100-103.

4. Catley BJ, Ramsay A, Servis C. Observations on the structure of the fungal extracellular polysaccharide, pullulan. Carbohydr Res. 1986;153:79-86.

5. Catley BJ. Utilization of carbon sources by Pullularia pullulans for the elaboration of extracellular polysaccharides. Appl Microbiol. 1971;22(4):641-649.
6. Deepak Kumar, Nisha Saini, Vinay Pandit, et al. An insight to pullulan: a biopolymer in pharmaceutical approaches. International Journal of Basic and Applied Sciences. 2012;1(3):202-219.

7. Thirumavalavar KTR, Manikkandani TR, Dhanasekar. Batch fermentation kinetics of pullulan from aureobasidium pullulans using low cost substrates. Biotechnology. 2008;7(2):317-322.

8. Gniewosz M, Sobczak E, Wojciechowska D, et al. Improvement of aureobasidium pullulans A.P.-3 for pullulan biosynthesis by associated mutagenesis. Pol J Food Nutr. Sci. 1999;8:235-243.

9. Paulraj Kanmani, Seung Taik Lim. Development and characterization of novel probiotic-residing pullulan/starch edible films. Food Chemistry. 2013;141:1041-1049.

10. Xiao Q, Lim L T, Tong Q. Properties of pullulan-based blend films as affected by alginate content and relative humidity. Carbohydrate polymers. 2012:87: 227-234.

11. Roukas T, Biliaderis CG. Evaluation of carbon pod as a substrate for pullulan production by Aureobasidium pullulans in an airlift reactor. Appl Biotechnol. 1995;80(1):77-89.

12. Opinion of the scientific panel on food additives, flavourings, processing aids and materials in contact with food on a request from the commission related to pullulan PI-20 for use as a new food additive. EFSA Journal. 2004;85:1-32.

13. Yoshifumi Murata, Takashi Isobe1, Kyoko Kofuji, et al. Preparation of fast dissolving films for oral dosage from natural polysaccharides. Materials. 2010;3;4291-4299.

14. Yue Li, Wallace Yokoyama, Jia Wu, et al. Properties of edible films based on pullulan-chitosan blended film-forming solutions at different $\mathrm{pH}$. RSC Adv. 2015;5:105844-105850.

15. Corniello C. Quick dissolving strips: from concept to commercialization. Drug Del Technol. 2006;6:68-71.

16. Vollmer U, Galfetti P. Rapid film: Oral thin films as an innovative drug delivery System and dosage form. Drug Dev Report. 2006;64-67. 